\title{
TÍNH CHẤT QUANG PHI TUYẾN KẾT HỢP TRONG VẠT LIỆU GỐM THỦY TINH GERMANOTELLURITE ÁP NHIẸT ĐIỆN
}

\author{
LỘ NHẬT TRƯỜNG \\ Khoa Công nghệ Hóa học, Truờng Đại học Công nghiệp Thành phố Hồ Chi Minh \\ lonhattruong@iuh.edu.vn
}

Tóm tắt. Vật liệu composite gốm thủy tinh dựa trên nền tảng vật liệu germanotellurite pha tạp bạc ô xít được áp nhiệt điện nhằm khảo sát khả năng kết hợp tính chất quang phi tuyến của thành phần gốm và lớp cấu trúc bề mặt biến tính của thành phần thủy tinh. Tính chất quang phi tuyến của ba loại vật liệu thủy tinh germanotellurite pha tạp bạc ô xít áp nhiệt điện, composite gốm thủy tinh và composite gốm thủy tinh áp nhiệt điện được khảo sát dựa trên cơ chế điều hòa bậc hai trong cùng điều kiện thực nghiệm. Kết quả chứng tỏ khả năng kết hợp hai phương pháp nhằm cải thiện đặc tính quang học đặc biệt của vật liệu này.

Từ khóa. Quang phi tuyến, thủy tinh, gốm thủy tinh

\section{COMBINATION OF NONLINEAR OPTICAL PROPERTIES IN THERMAL POLED GERMANOTELLURITE GLASS CERAMICS}

\begin{abstract}
Glass ceramic composite materials based on germanotellurite system doped with silver oxide has been thermally poled to investigate the possibility of combining nonlinear optical properties of ceramic components and the modified structural surface layer of glass component. Nonlinear optical properties of three types of samples: thermally poled doped silver oxide doped germanotellurite glass materials, ceramic glass composite and thermally poled glass ceramics composite of the same system are investigated based on Second Harmonic Generation in the same experimental conditions. The results demonstrate the ability to combine two methods to improve the special nonlinear optical properties of this materials.
\end{abstract}

Keywords. Nonlinear optics, germanotellurite glass, glass ceramics

\section{GIỚI THIỆU}

Nhu cầu sử dụng của các thiết bị quang phi tuyến như hệ thống chuyển đổi tần số và công tắc quang tốc độ cao đang ngày càng tăng lên và việc tích hợp các loại thủy tinh nền tellurite vào các thiết bị đó đang được nghiên cứu sâu. Những loại thủy tinh này có nhiều ưu điểm về sự ổn định hóa học kết hợp với dải bước sóng trong suốt rộng. Ngoài ra, trong lĩnh vực quang phi tuyến, loại vật liệu này cũng thu hút được sự chú ý nhờ vào chỉ số khúc xạ cao và độ nhạy điều hòa bậc ba lớn $[1,2]$. Việc kết hợp thủy tinh nền tellurite và các đảo tinh thể để tạo ra cấu trúc composite gốm thủy tinh đã cho thấy sự cải tiến đáng kể về mặt quang học. Các nghiên cứu trước đây cho thấy tiềm năng cải thiện tính chất trong suốt của loại vật liệu composite gốm thủy tinh nền tellurite bằng cách giảm chênh lệch chiết suất giữa nền và các đảo tinh thể dẫn tới mức tổn thất tán xạ thấp tại vị trí biên giới hạt và nền vô định hình [3-5]. Bên cạnh đó, các thành quả thu được từ phương pháp áp nhiệt điện cũng cho thấy tiềm năng cải thiện mạnh tính chất quang phi tuyến của các loại vật liệu thủy tinh thuần mà không làm mất đi tính chất trong suốt vốn là ưu điểm của loại vật liệu vô định hình này [6-8].

Gần đây, Tiến sĩ Lộ Nhật Trường và cộng sự đã phát minh thành công một loại vật liệu nanocomposite gốm thủy tinh dựa trên nền thủy tinh Germanotellurite. Cấu trúc germanotellurite mang đến sự kết hợp của nền tellurite và $10 \%$ ô xít germanium giúp cải thiện độ trong và độ chênh lệch của nhiệt độ chuyển hóa thủy tinh và nhiệt độ kết tinh. Yếu tố này quan trọng để giúp cho quá trình kéo sợi thủy tinh trong quá trình chế tạo các loại sợi quang học [4]. Bên cạnh đó, bằng cách thêm bạc ô xít để tạo ra cấu trúc [(100-x) $\left(70 \mathrm{TeO}_{2}\right.$ $\left.\left.10 \mathrm{GeO}_{2}-10 \mathrm{~K}_{2} \mathrm{O}-10 \mathrm{Nb}_{2} \mathrm{O}_{5}\right)+\mathrm{xAg}_{2} \mathrm{O}\right](\mathrm{x}=6 \mathrm{~mol} \%)$ và sau đó xử lý nhiệt, composite gốm thủy tinh với các đảo tinh thể phân bố đều bên trong lòng khối vật liệu (volume crystallization) đã được tạo ra thành công [9]. Sự kết tụ các nguyên tử bạc xảy ra trong quá trình xử lý nhiệt trở thành mầm tạo hạt bên trong các vùng pha phân tách. Quá trình kết tinh xảy ra bên trong vùng pha phân tách nằm lẫn bên trong khối vật liệu giúp cải thiện khả năng kết tinh khối thay vì chỉ xảy ra trên bề mặt vật liệu [9]. Đây là sự cải tiến lớn nếu so với 
kết quả thử nghiệm đối với vật liệu germanotellurite không pha tạp với bạc ô xít [4]. Cơ chế mới này làm cho Germanotellurite trở nên thú vị để nghiên cứu sâu hơn cả về tính chất hóa lý lẫn ứng dụng trong các thiết bị quang học.

Hiệu ứng quang điện điều hòa bậc hai có thể được tạo ra bằng phương pháp kết hợp gốm và thủy tinh [10] hoặc dung phương pháp áp điện. Vật liệu vô định hình như thủy tinh sẽ không mang tính chất quang phi tuyến điều hòa bậc hai do tính đối xứng vĩ mô của nó. Phương pháp áp điện giúp thay đổi cấu trúc đối xứng tại bề mặt của vật liệu vô định hình bằng cách áp một hiệu điện thế lớn lên tới hàng nghìn vôn trong quá trình gia tăng nhiệt độ giúp cho các ion nguyên tử được sắp xếp lại và tạo thành lưỡng cực cục bộ [6]. Lớp cấu trúc lưỡng cực này có bề dày từ vài nanomét đến vài micromét sẽ phá vỡ tính chất đối xứng của vật liệu vô định hình dẫn đến tính chất quang phi tuyến điều hòa bậc hai khá lớn. Các thử nghiệm áp nhiệt điện trên một số vật liệu có cấu trúc thủy tinh nền tellurite đã cho những kết quả tích cực [11]. Tuy nhiên, chưa có nghiên cứu nào về sự kết hợp của cả hai phương pháp đến hiệu quả cải thiện hiệu ứng quang điện điều hòa bậc hai trên nền vật liệu tellurite này.

Trong nghiên cứu này, chúng tôi muốn khảo sát khả năng cải thiện tính chất quang phi tuyến điều hòa bậc hai của vật liệu composite gốm thủy tinh nền germanotellurite pha tạp bạc ô xít khi kết hợp với phương pháp áp nhiệt điện. Các kết quả đo tính chất quang phi tuyến của chỉ riêng composite gốm thủy tinh và của riêng loại thủy tinh đó khi được áp nhiệt điện sẽ được đem đi so sánh với kết quả thu được của cùng loại vật liệu được áp dụng cùng lúc hai phương pháp. Sự bổ sung về cường độ và vị trí hoạt động của tính chất quang phi tuyến sẽ được dung để đánh giá hiệu quả của quá trình kết hợp hai loại phương pháp trên cùng một nền vật liệu.

\section{THƯC NGHIẸM}

Thủy tinh germanotellurite có thành phần $(100-\mathrm{x})\left[70 \mathrm{TeO}_{2}-10 \mathrm{GeO}_{2}-10 \mathrm{Nb}_{2} \mathrm{O}_{5}-10 \mathrm{~K}_{2} \mathrm{O}\right]-\mathrm{xAg}_{2} \mathrm{O}(\mathrm{mol} \%)$ $(\mathrm{x}=6 \mathrm{~mol} \%)$ được điều chế theo phương pháp truyền thống. Một hồn hợp gồm các loại bột thương mại độ tinh khiết cao $\mathrm{TeO}_{2}\left(99,99 \%\right.$, Alfa Aesar), $\mathrm{GeO}_{2}$ (> 99,99\%, Aldrich), $\mathrm{Nb}_{2} \mathrm{O}_{5}$ (99,9985\%, Alfa Aesar), $\mathrm{K}_{2} \mathrm{CO}_{3}\left(99,997 \%\right.$, Alfa Aesar) và lượng bạc nitrat (99,995\%, Alfa Aesar) đã được nấu chảy ở $900^{\circ} \mathrm{C}$ sau khi giữ ở nhiệt độ $150^{\circ} \mathrm{C}$ để loại bỏ hơi nước. Hỗn hợp nóng chảy được giữ ở nhiệt độ cao này trong 30 phút để phân hủy tất cả gốc nitrat và cacbonat. Sau đó, chất lỏng được rót vào đĩa bạch kim nung nóng ở $300^{\circ} \mathrm{C}$ trước khi được đưa vào một lò thứ hai để ủ ở cùng nhiệt độ $\left(300^{\circ} \mathrm{C}\right)\left(\sim \mathrm{Tg}-50^{\circ} \mathrm{C}\right)$, trong 6 giờ [9]. Tất cả các mẫu được đánh bóng bởi máy mài và giấy nhám nhiều cấp độ hạt khác nhau để thu được các mẫu có bề dày $1 \mathrm{~mm}$ đạt mức trong suốt quang học.

Tính chất nhiệt của mẫu thủy tinh sau khi điều chế bao gồm nhiệt độ chuyển hóa thủy tinh lẫn nhiệt độ kết tinh sẽ được nghiên cứu bằng thiết bị phân tích nhiệt lượng quét vi sai (DSC) thay vì các loại phân tích nhiệt vi sai (DTA) do các kết quả nghiên cứu trước cho thấy các giá trị nhiệt độ này đều nằm trong khoảng dưới $600^{\circ} \mathrm{C}$, thuộc giới hạn của các thiết bị phân tích nhiệt thuộc nhóm DSC. Khối lượng mẫu được kiểm soát trong phạm vị $30-40 \mathrm{mg}$ và tốc độ gia nhiệt là $20^{\circ} \mathrm{C} /$ phút.

Mẫu vật liệu composite gốm thủy tinh thu được bằng chu trình nhiệt một bước thay vì chu trình nhiệt hai bước (bước một ở lân cận nhiệt độ chuyển hóa thủy tinh và bước hai ở nhiệt độ kết tinh) thông dụng do ưu điểm về kích thước hạt cũng như tính chất quang phi thuyến tuyến tính (độ trong suốt). Thủy tinh được nâng thẳng lên gần với đỉnh nhiệt độ kết tinh (thấp hơn vài chục độ so với kết quả thu được thì kết quả phân tích nhiệt DSC) và giữ tại đó trong 15 phút.

Cấu trúc vô định hình của các mẫu thủy tinh cũng như pha tinh thể trong các mẫu composite gốm thủy tinh được điều chế sau đó được khảo sát bằng phương pháp nhiễu xạ tia X (XRD). Máy nhiễu xạ hiệu Philips X'PERT APD sử dụng bức xạ $\mathrm{Cu} \mathrm{K} \alpha$ với bước nhỏ $0,017^{\circ}$. Thời gian cho mỗi bước lên đến 59 giây. Giới hạn góc quay 2 theta từ $10^{\circ}$ đến $70^{\circ}$.

Phương pháp áp nhiệt điện được thực hiện trong không khí ở áp suất khí quyển. Sơ đồ thí nghiệm được thể hiện trên Hình 1. Một tấm wafer silicon và thủy tinh borosilicate chưa được đánh bóng đã được sử dụng tương ứng tại cực dương và cực âm. Silicon wafer rất hữu ích để tạo ra một bề mặt phẳng và do đó có khoảng cách đồng đều đến điện cực anốt. Các mẫu vật liệu thủy tinh pha tạp bạc ô xít (7T1G6Ag) được làm nóng đến $280^{\circ} \mathrm{C}$ và giữ trong 10 phút để ổn định nhiệt, điện thế phân cực được áp trong 30 phút. Sau đó nhiệt độ của mẫu được cho giảm xuống để ổn định cấu trúc trước khi tắt hiệu điện thế để loại bỏ điện trường. 


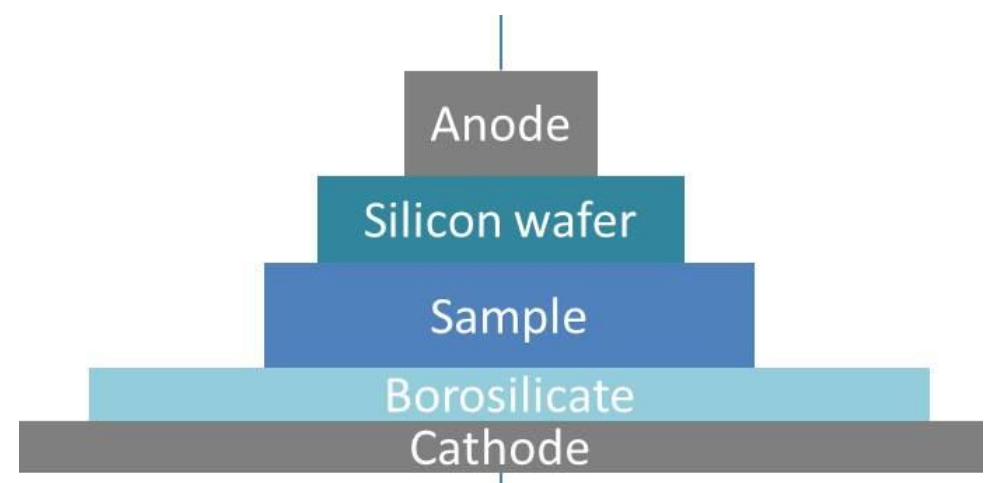

Hình 1: Thiết kế của phương pháp áp nhiệt điện

Tín hiệu quang phi tuyến điều hòa bậc hai của các mẫu được đánh giá bằng thiết bị được phát triển bởi Viện Khoa học Phân tử (ISM) tại Bordeaux, Pháp. Thiết bị bao gồm nguồn phát tia laser 1064nm phân cực được cho chiếu qua mẫu và sau đó tín hiệu đầu ra ở bước sóng ngắn (532nm) sẽ được tổng hợp và phân tích sau khi loại bỏ dòng bức xạ nền ở bước song 1064nm. Cấu trúc của thiết bị cụ thể như sau: ban đầu, chùm tia tới được tập trung vào mẫu với góc tới thay đổi được và tín hiệu sóng điều hòa bậc hai truyền ra được lọc tách để tạo ra tín hiệu nâng tần tại bước sóng 532nm. Cường độ sóng điều hòa bậc hai được ghi lại so với góc quay quanh trục thẳng đứng của mẫu $(\theta)$ trong mối tương quan với hướng tới của chùm laser kích thích ban đầu (Hình 2).

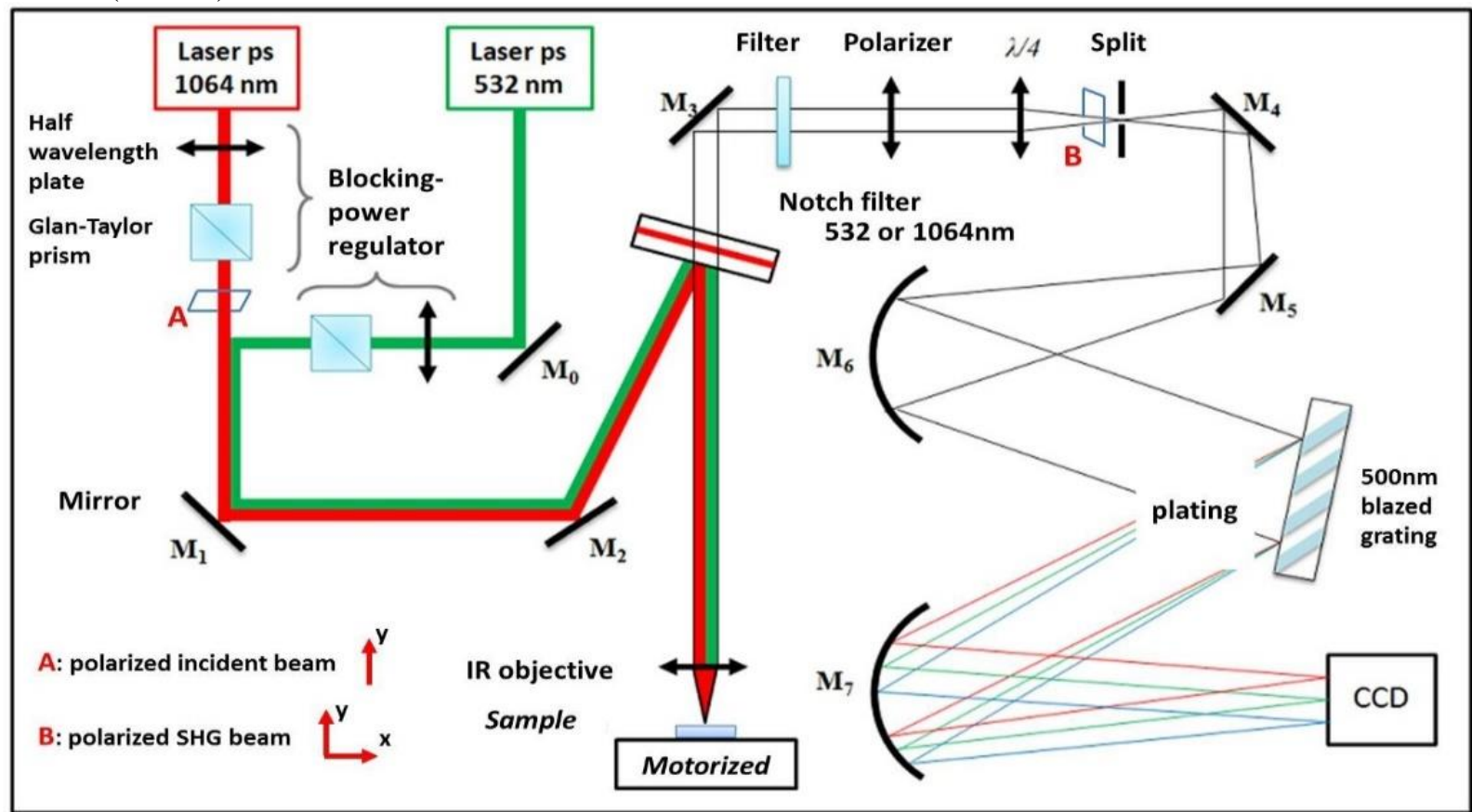

Hình 2: Thiết kế của máy phân tích tín hiệu nâng tần bậc hai [12]

\section{KẾT QUẢ VÀ BÀN LUẬN}

Đường cong DSC của mẫu thủy tinh được thể hiện trên hình 3 . Từ kết quả này, nhiệt độ chuyển hóa thủy tinh được xác định vào khoảng $350^{\circ} \mathrm{C}$, trong khi nhiệt độ kết tinh thứ nhất vào khoảng $450^{\circ} \mathrm{C}$. Dựa vào kết quả này, chúng tôi chọn nhiệt độ $480^{\circ} \mathrm{C}$ để chế tạo mẫu composite gốm thủy tinh vì nhiệt độ này cao hơn nhiệt độ bắt đầu kết tinh lần thứ nhất nhưng thấp hơn nhiệt độ bắt đầu kết tinh thứ hai $\left(\sim 550^{\circ} \mathrm{C}\right)$. Việc này giúp tăng tốc độ hình thành tinh thể, nhờ đó tăng khả năng kiểm soát kích thước tinh thể hóa trong mẫu composite. 


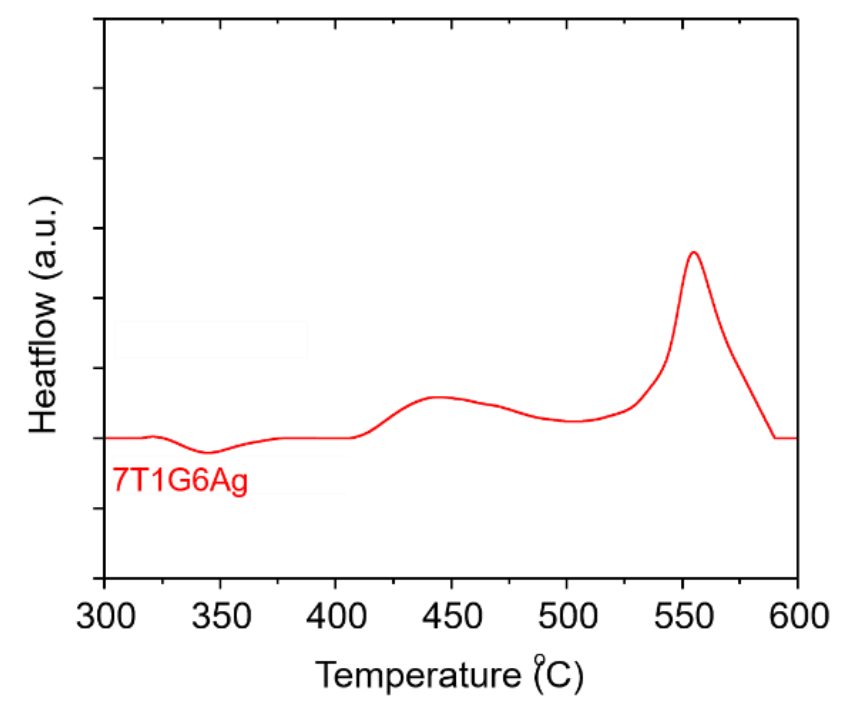

Hình 3: Đường cong DSC của mẫu thủy tinh germanotellurite

Kết quả đo nhiễu xạ tia $X$ của mẫu thủy tinh và mẫu composite gốm thủy tinh được cung cấp trong hình 4. Hình XRD phía trên cho thấy bằng chứng của mẫu thủy tinh khi các đỉnh nhiễu xạ ở dạng tù đặc trưng của các mẫu vật liệu vô định hình. Trong khi đó, các đỉnh nhiễu xạ XRD bên dưới phù hợp với cấu trúc pha $\mathrm{K}\left[\mathrm{Nb}_{1 / 3} \mathrm{Te}_{2 / 3}\right]_{4.8}$. Kết quả này tương đồng với các công bố trước đây trong cùng một họ thủy tinh germanotellurite.

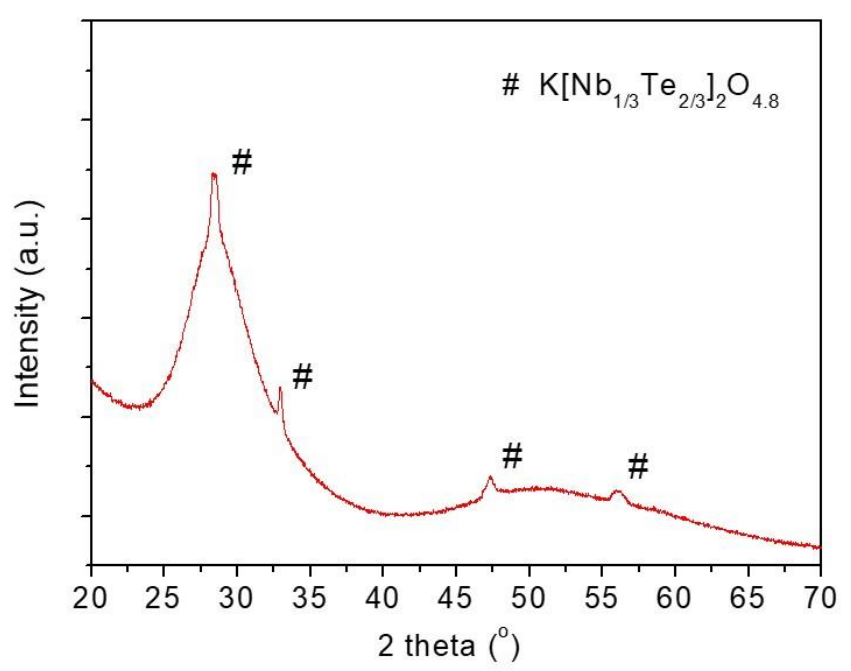

Hình 4: Các đỉnh nhiễu xạ tia $X$ của mẫu vật liệu composite gốm thủy tinh

Các nghiên cứu trước đây cho thấy chúng ta hoàn toàn có thể kiểm soát tính chất quang phi tuyến điều hòa bậc hai của các vật liệu composite bằng cách điều khiển kích thước hoặc mức độ phân bố của các đảo tinh thể trên nền thủy tinh. Kích thước càng lớn hoặc phân bố càng nhiều sẽ tương ứng với hiệu quả chuyển đổi tần số càng cao $[9,12]$. Tuy nhiên, nhằm khảo sát khả năng kết hợp tính chất quang phi tuyến tạo ra từ đảo tinh thể và bề mặt biến tính nhờ phương pháp áp nhiệt điện, kích thước của các đảo tinh thể được lựa chọn sao cho phù hợp với khả năng chuyển đồi tần số của phương pháp áp nhiệt điện. Phương pháp đó cũng như cường độ chùm laser đầu vào cũng được giữ không đổi trong suốt quá trình đo cả ba mẫu vật liệu nhằm tạo thuận tiện cho việc so sánh. Kết quả đo thủy tinh germanotellurite áp nhiệt điện được biểu diễn trên Hình 5. Trục tung thể hiện cường độ của tín hiệu điều hòa bậc hai (hay tín hiệu quang ứng với bước sóng 532nm), trong khi đó trục hoành đại diện cho góc lệch của chùm tia tới và mặt phẳng mẫu. Vì mẫu được quay xung 
quanh một trục xuyên tâm nên định hướng của các liên kết bề mặt sẽ thay đổi dẫn tới biến đổi về tính chất quang phi tuyến.

Đồ thị cho thấy sự tương đồng với các nghiên cứu trước đây với các loại vật liệu khác như hệ $\mathrm{SiO}_{2}$. $\mathrm{Khi}$ áp nhiệt điện, bề mặt mẫu sẽ xảy ra sự tái cấu trúc của các nguyên tử dẫn đến sự thay đổi về định hướng liên kết. Thủy tinh có cấu trúc vô định hình đặc trưng, nghĩa là đẳng hướng. Tuy nhiên, dưới tác động của điện trường giữa a nốt và ca tốt, các ion bên trong thủy tinh sẽ dịch chuyển theo hướng điện trường. Có một số nghiên cứu cho thấy các ion nhỏ sẽ có xu hướng dịch chuyển nhiều hơn như $\mathrm{K}^{+}$hay $\mathrm{Na}^{+}$[6]. Tuy vậy, vì các định hướng liên kết này có xu hướng song song với chùm tia tới nên hiệu ứng quang phi tuyến chỉ có thể quan sát thấy rõ khi chùm tia này tạo được góc tới lớn so với hướng liên kết. Kết quả cho thấy cường độ điều hòa bậc hai lớn nhất thu được tại góc $45^{\circ}$.

Dựa vào kết quả đo phía trên, sẽ là có lý nếu chúng ta chọn lựa loại composite cho ra tín hiệu quang phi tuyến có cường độ tương đồng. Chu trình nhiệt một bước ứng với quãng thời gian lưu nhiệt 15 phút tại $480^{\circ} \mathrm{C}$ là phù hợp nhất để so sánh với mẫu áp nhiệt điện thu được.

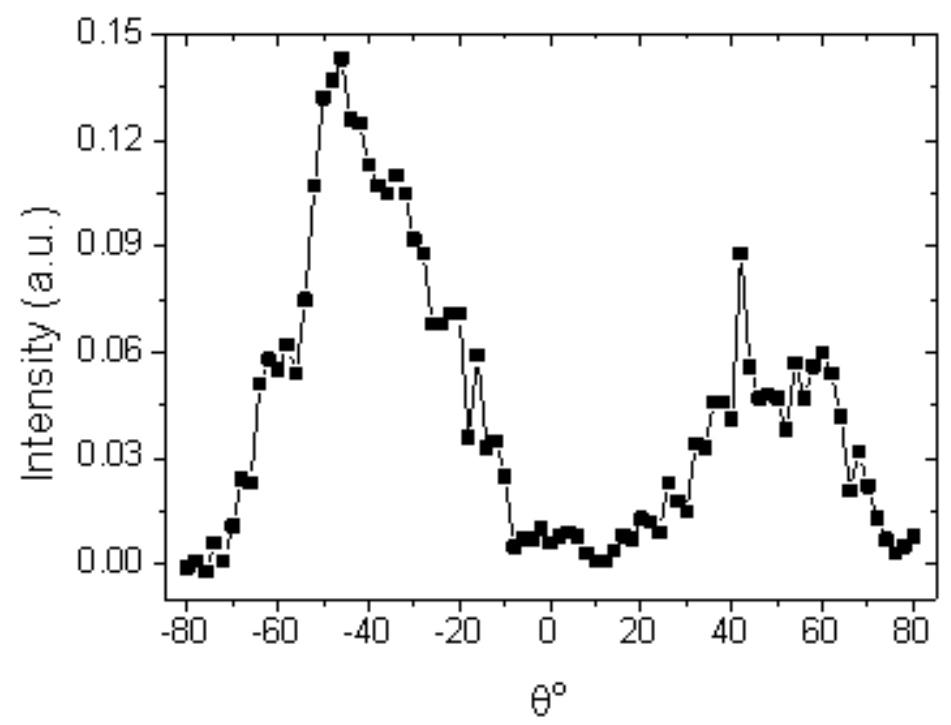

Hình 5: Tín hiệu quang phi tuyến điều hòa bậc hai của mẫu thủy tinh pha tạp $6 \%$ bạc ô xít

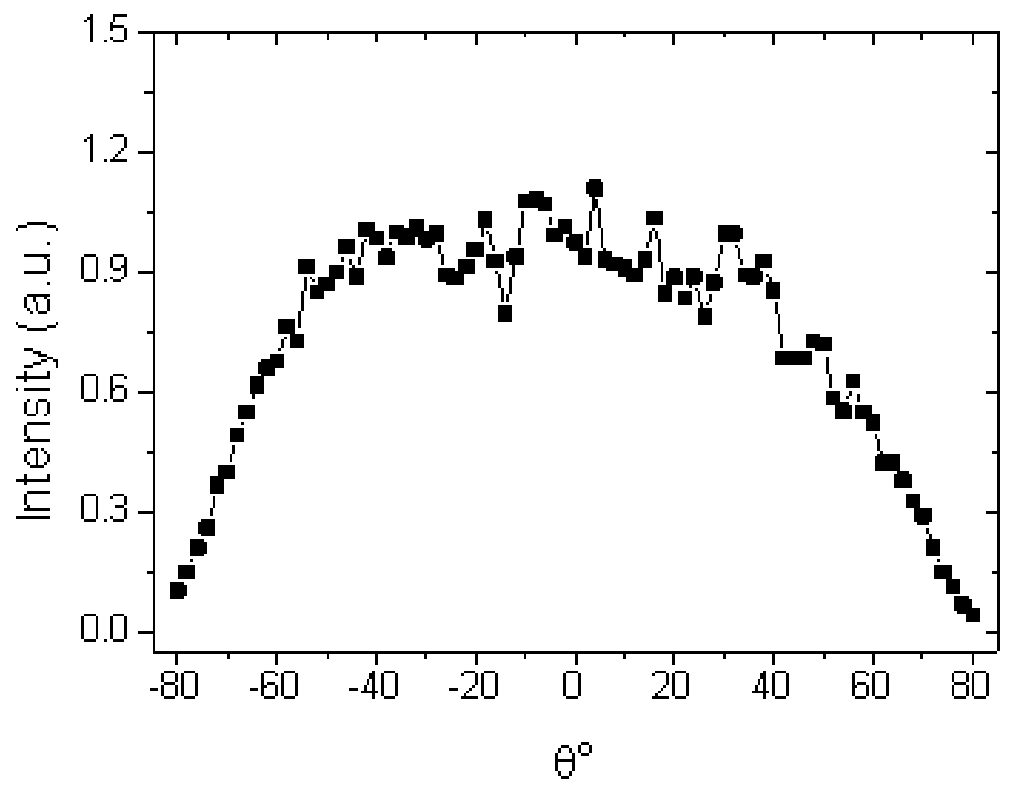

Hình 6: Tín hiệu quang phi tuyến điều hòa bậc hai của mẫu composite gốm thủy tinh gemanotellurite 
Kết quả đo vật liệu composite gốm thủy tinh germanotellurite được cung cấp trên Hình 6 cho thấy rõ sự đẳng hướng của tín hiệu quang phi tuyến điều hòa bậc hai. Đỉnh cao nhất ứng với góc lệch ít nhất $\left(0^{\circ}\right)$ của mẫu vật liệu so với góc phân cực của chùm laser tới. Khi góc lệch tăng dần thì cường độ tín hiệu tần số chuyển đổi giảm dần và tiến về không khi góc lệch gần như là vuông góc. Sự suy giảm này do ảnh hưởng bởi quãng đường đi của chùm tia bên trong khối vật liệu thay đổi đáng kể khi xoay mẫu hơn $45^{\circ}$. Kết quả tương đồng có thể tìm thấy trong các nghiên cứu khác [13] và hiện tượng này cũng có thể quan sát thấy trong Hình 5 với góc tới lớn hơn $45^{\circ}$.

Kết quả đo composite gốm thủy tinh germanotellurite áp nhiệt điện cho thấy có sự biến đổi cơ bản hình thái của tín hiệu theo góc tới. Hình dạng đặc trưng của composite gốm thủy tinh còn khá rõ đặc biệt gần với góc lệch nhỏ (Hình 7). Tuy nhiên, tại góc nghiêng $30-45^{\circ}$ có dấu hiệu của sự chồng tín hiệu khi cường độ ánh sáng $532 \mathrm{~nm}$ thu được cao hơn so với vùng góc tới nhỏ. Vị trí của sư gia tăng tín hiệu điều hòa bậc hai cũng tương đồng với vị trí cường độ cực đại của mẫu thủy tinh áp điện không chứa tinh thể. Đây là bằng chứng chứng tỏ khả năng kết hợp của hai loại phương pháp chế tạo vật liệu có tính chất quang phi tuyến, mở ra triển vọng vận dụng được ưu điểm của từng loại phương pháp. Khi dùng gốm thủy tinh, tính chất quang phi tuyến càng cao thường phải trả giá bằng độ trong suốt giảm, trong khi vật liệu áp điện lại thể hiện sự phụ thuộc quá lớn vào góc tới của chùm laser kích thích.

Tuy nhiên, ảnh hưởng điện trường lên bề mặt vật liệu thủy tinh nền germanotellurite hay tác động của các hạt tinh thể lên hiệu quả thay đổi liên kết bề mặt của điện trường còn chưa được khảo sát kỹ lưỡng nhằm giải thích đầy đủ hơn cơ chế tổng hợp tín hiệu quang phi tuyến điều hòa bậc hai. Trong các nghiên cứu tiếp theo, chúng ta có thể áp dụng thêm nhiều phương pháp phân tích cấu trúc để làm rõ hơn về vấn đề này.

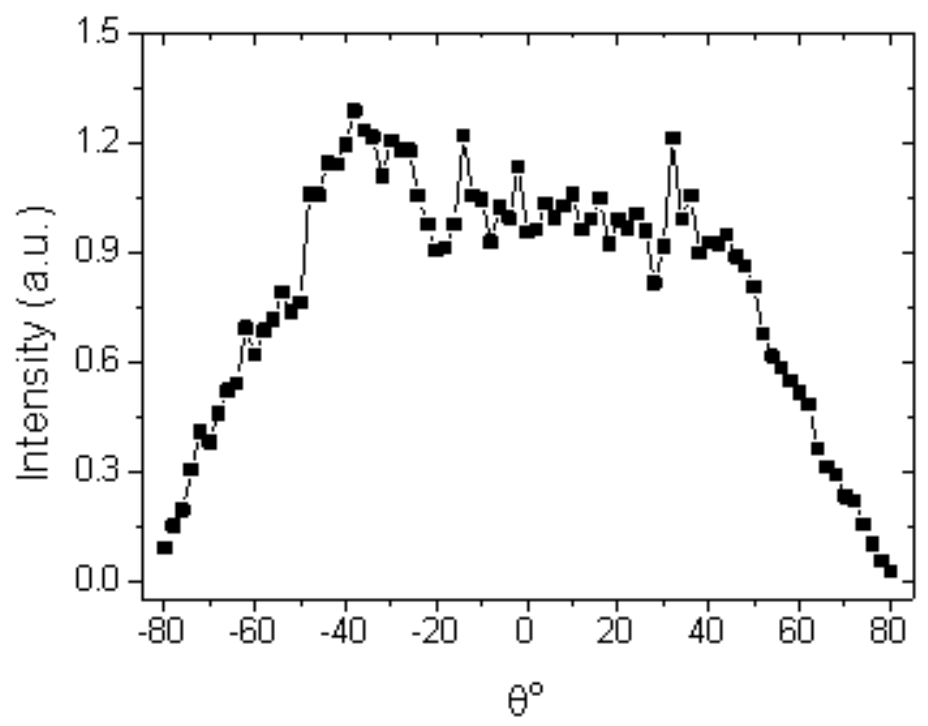

Hình 7: Tín hiệu quang phi tuyến điều hòa bậc hai khi áp dụng phương pháp áp nhiệt điện cho mẫu composite gốm thủy tinh

\section{KẾT LUÂN}

Nghiên cứu cho thấy bằng chứng về khả năng cải thiện tính chất quang phi tuyến khi kết hợp đồng thời các đảo tinh thể lẫn bên trong khối vật liệu và bề mặt biến đổi do phương pháp áp nhiệt điện tạo ra. Kết quả đo quang phi tuyến của vật liệu composite gốm thủy tinh sau khi áp nhiệt điện cho thấy các đỉnh tín hiệu quang điều hòa bậc hai tại những vị trí ứng với thủy tinh cùng loại được áp nhiệt điện mà không có sự hiện diện của đảo tinh thể.

\section{LÒ̀I TRI ÂN}

Tác giả (TS. Lộ Nhật Trường) muốn bày tỏ lòng biết ơn tới Quỹ Phát triển Khoa học \& Công nghệ Quốc gia (NAFOSTED), Trường Đại học Công nghiệp thành phố Hồ Chí Minh và trường đại học Bordeaux đã hỗ trợ tài chính hoặc trang thiết bị cho nghiên cứu này. 


\section{THAM KHẢO}

[1] El-Mallawany, Raouf AH. Tellurite glasses handbook: physical properties and data. CRC press, 2016.

[2] Ferreira EA, Cassanjes FC, Poirier G. Crystallization behavior of a barium titanate tellurite glass doped with Eu3+ and Er3+. Opt Mater., vol. 35, pp. 1141-1145, 2013.

[3] Jain H. Transparent ferroelectric glass-ceramics. Ferroelectrics, vol. 306, pp. 111-127, 2004;.

[4] Monteiro G, Santos LF, Pereira J, Almeida RM. Optical and spectroscopic properties of germanotellurite glasses. J Non-Cryst Solids, vol. 357, pp. 2695-2701, 2011.

[5] Monteiro G, Santos LF, Almeida RM, D'Acapito F. Local structure around Er3+ in GeO2-TeO2-Nb2O5-K2O glasses and glassceramics. J Non-Cryst Solids. Vol. 377, pp. 129-136, 2013.

[6] Dussauze, Marc, et al. Large second-harmonic generation of thermally poled sodium borophosphate glasses. Optics express, vol. 13, pp. 4064-4069, 2005.

[7] Narazaki, Aiko, et al. Induction and relaxation of optical second-order nonlinearity in tellurite glasses. Journal of applied physics, vol. 85, pp. 2046-2051, 1999.

[8] Lasbrugnas, Carine, et al. Second harmonic generation of thermally poled tungsten tellurite glass. Optical Materials, vol. 31, pp. 775-780, 2009.

[9] Truong, Lo Nhat, et al. Second harmonic generation in germanotellurite bulk glass-ceramics. Journal of the American Ceramic Society, vol. 100, pp. 1412-1423, 2017.

[10] Truong LN, Dussauze M, Fargin E, et al. Isotropic octupolar second harmonic generation response in LaBGeO5 glass-ceramic with spherulitic precipitation. Appl Phys Lett., vol. 106, pp. 161901, 2015.

[11] Lasbrugnas, C., Thomas, P., Masson, O., Champarnaud-Mesjard, J. C., Fargin, E., Rodriguez, V., \& Lahaye, M. Second harmonic generation of thermally poled tungsten tellurite glass. Optical Materials, vol. 31, pp. 775-780, 2009. [12] Crémoux, T., "Physico-chimie aux interfaces de systèmes vitreux à charge d'espace". Université Sciences et Technologies-Bordeaux I, 2013.

[13] Vigouroux H, Fargin E, Gomez S, et al. Synthesis and multiscale evaluation of LiNbO3-containing silicate glassceramics with efficient isotropic SHG response. Adv. Funct. Mater. vol. 22, pp. 3985-3993, 2012.

Ngày nhận bài: 12/09/2019

Ngày chấp nhận đăng: 17/06/2020 